\title{
Intervenções Psicológicas na Psico-Oncologia
}

\author{
Elisa Maria Parabyba Campos* \\ Avelino Luiz Rodrigues** \\ Pablo Castanbo**
}

\section{Resumo}

O objetivo deste trabalho é divulgar a Psico-Oncologia como uma área de conhecimento que ampliou as possibilidades de atendimento ao portador de câncer seu familiar e equipe de saúde. O surgimento da Psico-Oncologia é influenciado por diversos fatores relacionados ao desenvolvimento das ciências, mas não deixa de ser uma necessidade que responde a uma demanda dos últimos anos, quando o câncer não para de atingir cada vez mais pessoas, levando as pesquisas a descobertas intrigantes, mas que muitas vezes não explicam o surgimento e a evolução da doença. A Psico-Oncologia nasce enfatizando os fatores psicossociais, biológicos e psicológicos no atendimento ao portador de câncer, e busca também uma compreensão maior dos processos de adoecimento e desenvolvimento da moléstia bem como as implicações na vida familiar dos portadores de câncer.

Palavras-chave: Psico-Oncologia, oncologia, psicoterapia

\section{Psychological Interventions in Psycho-Oncology}

\begin{abstract}
This work aims at divulging the main concepts of Psycho-Oncology as a new area of knowledge, involving the attendance of cancer patients, their families and health teams. Psycho-Oncology's creation was influenced by many different factors related to scientific development, but also constitutes an answer for a contemporary demand in face of an unprecedented number of new cases. This situation has conducted research to important discoveries, notwithstanding in many cases not being able to explain the causes nor the evolution of the disease. The reasons justifying this work involve the need for a systematized account accompanied of some necessary critical reflections, since information on the area is still scarce.
\end{abstract}

Keywords: Psycho-Oncology, oncology, psychotherapy 
We are not ourselves when nature, being oppressed, commands the mind to suffer with the body. William Shakespeare, King Lear

Aproximadamente há quatro décadas o psicólogo vem sendo solicitado para que faça parte de equipes multidisciplinares, para atuação em hospitais e outros equipamentos de promoção e atenção à saúde.

No entanto, só recentemente os cursos de graduação responsáveis pela formação profissional em Psicologia passaram a dedicar maior atenção a um fenômeno que vem ocorrendo em relação às áreas de atuação do psicólogo. É o advento de um processo de maior atenção à saúde, da criação de novas políticas de promoção de saúde e, portanto, da necessidade de profissionais com formação adequada para atuar nesta área.

Quando a Psicologia foi constituída como profissão, através da Lei $\mathrm{N}^{\circ}$ 4.119, de 27 de agosto de 1962, que dispõe sobre os cursos de formação em psicologia e a regulamentação da profissão de psicólogo (Conselho Federal de Psicologia, 2016), o seu artigo $13^{\circ}, \$ 1$, especificava as funções do psicólogo "a utilização de métodos e técnicas psicológicas com os seguintes objetivos: diagnóstico psicológico; orientação profissional; orientação psicopedagógica; solução de problemas de ajustamento"(Conselho Federal de Psicologia - CFP, 2016).

Evidentemente estes objetivos não levavam em conta algumas atividades que atualmente são desenvolvidas pelos profissionais de psicologia, tais como as práticas assistenciais junto a instituições de saúde como Unidades Básicas de Saúde (UBS), Centros de Atenção Psicossocial (CAPS) e Hospitais.

Uma rica história foi construída (Kerbauy, 2002; Gorayeb, 2010; Maia, Silva, Martins, \& Sebastiani,2015; Alves, Santos, Ferreira, Costa, \& Costa,2017). Muitos desafios foram impostos, como a superação do modelo clínico individual e a construção de um novo campo do saber, a psicologia da saúde. Acima de tudo houve uma imposição da realidade, o contexto da instituição de saúde contém diferenças significativas do locus operandi do psicólogo clínico e a prática assistencial psicológica passou por diferentes adaptações para corresponder àquilo que dela se esperava.

De acordo com Spink (2000), atuar na instituição de saúde muitas vezes vai colocar o profissional em contato com questões que vão além da subjetividade individual, criando uma interface entre a psicologia clínica e a psicologia social. Esta autora coloca a Psicologia da
Saúde como área da Psicologia Social. Embora não concordemos inteiramente com esta colocação, sem dúvida as instituições de saúde vão posicionar o psicólogo face a problemas de cunho eminentemente psicossocial. Nas casas de apoio, por exemplo, onde crianças portadoras de câncer são recebidas para aguardar intervenções médicas, realizadas em hospitais, um familiar acompanha a criança e tem de conviver com os familiares de outras crianças, surgem questões que frequentemente requerem uma visão e uma compreensão de psicologia grupal, além de social e cultural dos fatos, mais do que exatamente abordagens psicológicas individuais dos problemas.

Campos, Rodrigues, Machado e Alvarez (2007) em trabalho realizado em casa de apoio utilizaram uma técnica de grupo para trabalhar uma complexa situação surgida no cotidiano das mães destas crianças, que abandonaram suas tarefas na casa de apoio, inclusive, em relação aos próprios filhos em função de dificuldades emocionais, que emergiram como consequência do afastamento do domićlilo e de seus ambientes socioculturais, para acompanharem os filhos nos tratamentos.

Por outro lado, se observa na prática que intervenções em uma pessoa acometida por alguma enfermidade que atinge seu corpo, requer do profissional de psicologia posturas diferentes daquela adotada no atendimento a pacientes sem doenças orgânicas. A doença coloca o indivíduo em um estado de regressão que é maior quanto mais grave for a patologia, além disto, apresentam uma estrutura egóica fragilizada e com seus espaços mentais inundados pela angústia do adoecimento e todas as suas consequências.

$\mathrm{O}$ atendimento a pacientes portadores de câncer, seus familiares e equipes de saúde, reveste-se de características específicas que só irão se tornar evidentes no atendimento a este tipo de doente.

A primeira característica a ser apontada, é a extrema fragilidade na qual em geral os pacientes se encontram, a partir de um diagnóstico de neoplasia maligna. Ao mesmo tempo, o que se observa na maioria dos casos, é uma desestruturação da família, mesmo que momentaneamente, o que cria situações complexas a serem abordadas pelo profissional de psicologia.

Outro aspecto presente nos casos de portadores de câncer é a possibilidade de morte que isto representa. Mesmo que em alguns casos esta possibilidade seja remota, em função de desinformação, crenças e mitos que cercam o câncer, a morte é sempre um fantasma que permeia a realidade e as fantasias do paciente e seus familiares. 
Além do paciente e de seus familiares, as equipes de saúde que atendem pacientes portadores de câncer têm se revelado pacientes em potencial. O "stress" provocado pelo contato diário com a angústia, a dor, e o sofrimento dos pacientes, coloca os profissionais em um estado de alerta constante, que termina por desencadear uma série de sentimentos que podem prejudicar o equilíbrio emocional da equipe trazendo prejuízos consideráveis. $\mathrm{O}$ cuidado com o cuidador é hoje uma realidade, merecendo a atenção de diferentes áreas de profissionais de saúde.

A proposta, em síntese, da psico-oncologia, é estudar e prestar atendimento aos pacientes portadores de câncer, seus familiares e equipes de cuidadores, no que diz respeito às questões subjetivas relacionadas aos processos de adoecer oncológico. Diferentes questões psicológicas surgem neste processo de adoecer, desde o seu diagnóstico até a sua resolução, que pode vir com a sua remissão, mas, também, com a cronificação ou com a morte do paciente.

Os primeiros estudiosos dos aspectos emocionais presentes nos portadores de câncer, como LeShan (1984) e Simonton (1987), buscaram evidenciar a relação dos estados psicológicos com a evolução da doença.

A partir da evidência deste fato, visão esta aceita por muitos, mas não por todos os profissionais da área de saúde, um novo espaço se construiu. O espaço para os aspectos subjetivos na promoção e atenção à saúde oncológica, tanto no diagnóstico e tratamento como no prognóstico e na prevenção, conteúdos psicológicos e psicossociais têm a sua importância e devem, sempre, ser levados em consideração. A Psico-Oncologia trata deste espaço, desta instância na Oncologia.

$\mathrm{Na}$ antiguidade, Hipócrates (1999/ séculos IV a.C.) no texto "Da natureza do homem" propunha uma Medicina voltada para o ser humano como um todo, onde mente e corpo funcionariam harmonicamente, propiciando o bem-estar e a saúde. Para ele, o rompimento desta harmonia era o que fazia com que ocorresse a doença, em qualquer das suas manifestações. Naquele tempo estudava-se os "humores", que influenciariam o temperamento e sua relação com a saúde e a doença.

$\mathrm{Na}$ atualidade esta área é explorada, principalmente, pela Psiconeuroimunologia, que tem como um de seus objetivos o estudo das mudanças do sistema imunológico relacionados e concomitantes a determinados estados mentais, como, por exemplo, as depressões (Ader, Cohen \& Felten,1995).

A Psico-Oncologia nasce influenciada por diversos fatores pertinentes ao desenvolvimento das ciências, mas não deixa de ser uma necessidade que responde a uma demanda do século XX e XXI, quando o câncer apresenta uma prevalência crescente e pesquisas revelam descobertas intrigantes que muitas vezes não se explicam por razões unicamente biológicas.

\section{O Câncer}

Galeno (130-200 d.C.) foi quem construiu a primeira classificação dos tumores. Cabe ainda ressaltar que foi este mesmo personagem, quem associou estados melancólicos em mulheres com o surgimento de formações tumorais (Gimenez, 1994).

Segundo Coelho (1998), o câncer tem sua origem coincidente com a história do próprio homem e é uma doença relacionada aos seus hábitos, cultura, fatores ambientais e, acrescentaríamos, aspectos biológicos. Segundo esse autor, o primeiro a descrever a palavra "carcinos" e a definir o câncer como uma doença de mau prognóstico foi Hipócrates, em 500 a.C., ou seja, o câncer sempre existiu na humanidade, sendo que a descrição mais antiga do câncer vem do Egito em aproximadamente 3000 a.C. (Mandal, 2019).

$\mathrm{Na}$ verdade, mais de 200 doenças agrupam-se dentro da categoria que é denominada como câncer. Sua etiologia é complexa, sendo descrita como uma doença multifatorial e que surge a partir da mutação no código genético de apenas uma célula, que por sua vez se multiplica, dando origem a outras células anômalas que se agrupam gerando aquilo que será a massa tumoral (Schwartsmann, Katz, Cunha, Vinholes, Gansl, \& Simon, 1991).

Servan-Schreiber (2008), em trabalho autobiográfico após um câncer de cérebro, faz uma definição literária, mas verdadeira do processo de instalação de um câncer em um organismo:

Tomado pelo câncer, o organismo vive uma guerra total. As células cancerosas se comportam como bandos armados sem fé nem lei, liberados das imposições da vida em sociedade que caracterizam um organismo em boa saúde. Com seus genes anormais, elas escapam aos mecanismos de regulação dos tecidos. Perdem, por exemplo, a obrigação de morrer depois de um certo número de divisões, tornando-se, portanto, "imortais". Fazem como se não escutassem os sinais dos tecidos circundantes que, alarmados pela falta de espaço, lhe pedem incessantemente que parem de se multiplicar. Pior, estes se intoxicam pelas substâncias secretadas pelas células cancerosas. Esses venenos criam uma inflamação local que estimula ainda mais sua expansão em detrimento dos territórios 
vizinhos. Finalmente, como um exército em campanha que precisa assegurar seu abastecimento, as células cancerosas requisitam os vasos sanguíneos das proximidades e os obrigam a proliferar a fim de fornecer o oxigênio e os nutrientes indispensáveis ao crescimento do que vai rapidamente se tornar um tumor (p.43).

O câncer é uma das doenças de maior prevalência e incidência em todo mundo. Dados fornecidos pela Organização Mundial de Saúde informam que, anualmente, 15 milhões de pessoas são diagnosticadas como novos casos de câncer, o que resultou em 9.6 milhões de mortes em 2018 (OPAS Brasil, 2018)

A estimativa de incidência em 2018 segundo a OMS foi de 253.9 casos por 1000.00 habitantes, no Brasil 217.2. (International Agency for Research in Cancer, 2018)

É a segunda causa de morte no Brasil, perdendo apenas para as doenças cardiorrespiratórias. A estimativa para casos novos no ano 2020 são de $387.980 \mathrm{em}$ homens e 297.980 em mulheres (Instituto Nacional de Câncer, 2020) O custo anual total da doença em 2010 foi estimado em aproximadamente US $\$ 1,16$ bilhões de dólares americanos (Opas Brasil, 2018).

Ao se diagnosticar um câncer, dizer que um tumor é maligno não é o suficiente. É preciso dizer qual o seu grau de agressividade, isto é, o grau de invasibilidade em tecidos adjacentes e outras características que são particulares e distintivas.

Estas neoplasias têm a peculiaridade de criar uma rede de vasos sanguíneos a sua volta para que possam ser alimentados; a este quadro dá-se o nome de angiogênese e pode ocorrer em outras situações clínicas. Uma das possibilidades de tratamento do câncer atualmente é impedir ou paralisar a criação desta rede de vasos para que o tumor não tenha do que ou como se alimentar.

Os tumores malignos têm ainda a característica de criarem tumor, os chamados cânceres metastáticos, que começaram em um órgão do corpo e se espalharam para outras partes do corpo (Instituto Oncoguia, 2020), podemos dizer que elas desprendem do tumor "primário" e através da corrente sanguínea ou dos vasos linfáticos se espalham pelo corpo.

O câncer não é uma doença de fácil diagnóstico. Além de uma anamnese bem-feita, pelo médico, bem como um exame clínico cuidadoso, exames complementares são necessários, pois podem confirmar o diagnóstico além de dar as características do tumor tais como sua agressividade e estadio, que é a avaliação da taxa de crescimento e a extensão da doença, mas também do tipo de tumor e sua relação com o organismo do paciente (Instituto Nacional de Câncer, 2020).

Pelo que apresentamos, adentrar em um hospital e participar das questões de saúde como no caso do câncer, requer uma formação complementar para o profissional de psicologia, além de uma reflexão de ordem social uma vez que vai ser colocado face a uma realidade dolorosa que desafia o poder público no sentido de encontrar as soluções possíveis para uma situação que exige uma rede de atendimento à população que infelizmente ainda não foi possível atingir. O câncer é um problema de saúde pública no Brasil e no mundo.

Atualmente são inúmeras as formas de se lutar contra um câncer, mas os tratamentos são em geral invasivos, com efeitos colaterais dolorosos e desconfortáveis, levando a alterações de comportamentos e dificuldades de adaptação com sofrimento psíquico que merecem a atenção de profissionais da saúde mental.

Não foi aí que se iniciaram, mas foi de fundamental importância os movimentos de humanização e de integralidade, que os aspectos subjetivos do paciente portador de câncer passaram a ser considerados como uma faceta importante a ser observada e cuidada.

Cabe à Psico-Oncologia o cuidado com os aspectos de natureza psicológica mobilizados pela doença e seus tratamentos. O que se observa é que a cada tratamento emergem comportamentos e sentimentos distintos, mas não só cada tratamento produz uma reação diferente, as características pessoais e sociais e culturais podem gerar distintas formas de enfrentar a doença.

\section{A Psico-Oncologia}

Diferentes fatos criaram um campo fértil para o surgimento da Psico-Oncologia como campo estruturado de saber, notadamente o desenvolvimento da psicologia, da psiquiatria, da medicina e, principalmente, as mais recentes formas de se pensar e tratar os doentes e suas doenças, como o da universalidade e integralidade, que conjugam as ações direcionadas à materialização da saúde como direito e como serviço (Pinheiro, 2009).

Há aproximadamente 40 anos essa área produziu um modelo no qual o campo psicológico foi integrado como uma subespecialidade da oncologia por sua criadora Jimmie Holland. No Brasil, a Psico-Oncologia é definida por Gimenes (1994) como uma interface entre a Psicologia e a Oncologia.

Um de seus objetivos principais, na ocasião, era a de substituir mitos, crenças e estigmas do portador de câncer, por conhecimento e mecanismos de enfrentamen- 
to melhor adaptados aos tratamentos, com a inclusão de grupos de autoajuda, suporte psicossocial, entre outros. A partir destes focos se tornou possível o estudo dos comportamentos psicológicos associados ao câncer, o desenvolvimento de formas de observação, compreensão e intervenção e de novas posturas frente ao tratamento.

Holland (2002) sublinha a importância da fundação do Memorial Sloan-Kettering Câncer Center, em 1980, ali inicia-se um trabalho que objetivava desenvolver estudos voltados para a descoberta de procedimentos psicológicos que ajudariam a enfrentar o câncer.

Se até então o portador de câncer era tratado apenas por oncologistas, a criação da interconsulta possibilitou uma abordagem global do paciente, onde além da intervenção medicamentosa, a figura do psicólogo era solicitada para ajudar a tratar de sintomas de angústia, depressão e ansiedade, entre outros. Logo em seguida se inicia a intervenção de apoio, por parte da psicologia, aos familiares, uma vez que muitas vezes a família do paciente passa por momentos de desestruturação, como assinalamos acima, como consequência da falta de informação, orientação e suporte em diferentes fases da doença.

Se quisermos datar formalmente o início da Psico-Oncologia, podemos nos reportar às palavras de Holland (2002), "beginnings of psycho onchology date to the mid1970 " (p.213), em artigo publicado com a finalidade de divulgar o início de um movimento que passou a mudar as atitudes e os conceitos a respeito do câncer.

A Psico-Oncologia procura dar aos profissionais de saúde em geral, às famílias envolvidas e à comunidade como um todo, uma nova visão sobre o câncer, uma possibilidade de compreensão do processo de adoecer, como consequência de fatores biopsicossociais, e propõe ainda a possibilidade de uma maior compreensão das respostas psicológicas ao adoecimento, aos tratamentos e, posteriormente, à reabilitação e à sobrevivência.

No Brasil, a partir de encontros sobre Psico-Oncologia realizados em 1989, que posteriormente transformaram-se em congressos, passou-se a estudar a Psico-Oncologia, criando uma definição da área. De acordo com Gimenes (1994): A Psico-Oncologia representa a área de interface entre a Psicologia e a Oncologia e utiliza o conhecimento educacional, profissional e metodológico proveniente da Psicologia da Saúde para aplicá-lo:

$1^{\circ}$. Na Assistência ao paciente oncológico, à sua família e aos profissionais de Saúde envolvidos com a prevenção, o tratamento, a reabilitação e a fase terminal da doença; $2^{\circ}$. Na pesquisa e no estudo de variáveis psicológicas e sociais relevantes para a compreensão da incidência, da recuperação e do tempo de sobrevida após o diagnóstico de câncer;

$3^{\circ}$. Na organização de serviços oncológicos que visem ao atendimento integral do paciente (físico e psicológico) enfatizando de modo especial a formação e o aprimoramento dos profissionais de Saúde envolvidos nas diferentes etapas do tratamento. (p.46)

A partir do $1^{\circ}$. Congresso Brasileiro de Psico-Oncologia realizado em São Paulo,1994 foi criada a Sociedade Brasileira de Psico-Oncologia, que congrega profissionais ligados à área, oferecendo cursos, assistência e treinamento a profissionais de saúde que trabalham com câncer.

\section{As possibilidades de intervenção}

\section{- Junto ao paciente}

As intervenções em Psico-Oncologia não diferem, em seus objetivos, das intervenções psicológicas em geral. Segundo Holland (1990) os primeiros profissionais da saúde que iniciaram o que posteriormente seria chamado de Assistência Psicológica foram as enfermeiras e as assistentes sociais. Em função da convivência diuturna com os pacientes estes profissionais foram se conscientizando da demanda por um tipo de assistência que pudesse auxiliar de alguma forma as diferentes reações psicológicas que foram surgindo conforme o desenvolvimento da doença.

Posteriormente Psiquiatras e Psicólogos passaram a fazer parte do grupo inicial em função das necessidades dos pacientes (Holland, 2002).

Vale sublinhar que o profissional de Psico-Oncologia, presta assistência a um paciente portador de câncer ou a seus familiares e ele deve dominar alguns conteúdos que não são obrigatórios em outros tipos de atendimento tais como alguns tipos de câncer, efeitos colaterais dos tratamentos, como no caso de uma Quimioterapia.

Uma das principais exigências a serem cumpridas, diz respeito ao conhecimento em relação ao câncer e aos tratamentos relacionados à doença. Importante frisar, que não estamos falando de um domínio ou submissão ao discurso médico, do oncologista em particular, mas de um conhecimento básico da doença, de seu desenvolvimento, de seus fatores de risco e etiológicos e dos respectivos tratamentos que se fazem necessários.

O psicólogo que trabalha junto a pacientes oncológicos tem a consciência, de forma particularmente acentuada, de que lida com experiências emocionais, de seus pacientes e familiares, às ameaças concretas, 
objetivas, uma angústia de morte calcada na realidade factual, e com ameaças simbólicas que, em grande proporção, contém uma intensa contaminação, daquelas experiências emocionais decorrentes das ameaças concretas e objetivas.

Qualquer atendimento ao paciente portador de câncer, deve ser voltado para técnicas integrativas, reconstrutivas e de suporte. Muitas vezes psicopedagógicas, de esclarecimento, aconselhamento e de acolhimento. Vale frisar que este tipo de paciente passa por uma perda de suas certezas e referenciais existenciais, que resulta em sentimentos de medo, ansiedade, tristeza e culpa (Grassi, Biancosino, Marmai, Rossi \& Sabato, 2007)

A literatura não prescreve um referencial teórico específico para o atendimento do paciente oncológico. O importante é que qualquer que seja a escuta do profissional, o manejo da sua intervenção seja marcado pela postura compreensiva e acolhedora. O entendimento da importância da existência de mecanismos defensivos e de "coping", por parte do paciente para se proteger das experiências emocionais de impotência, de incerteza, do desamparo, da angústia e da tristeza, também deve nortear a atuação do profissional.

Um aspecto importante é a depressão, que pode ocorrer em qualquer momento da doença. Citamos acima os estudos da psiconeuroimunologia (Ader, Cohen \& Felten, 1995) demonstrando a queda da atividade imunológica em estados depressivos, o que diminuiria o grau de defesa do organismo, possibilitando uma progressão da doença, outras pesquisas corroboraram tais achado, citamos Bauer (2004) e Deitos \& Gaspary (1997).

\section{Intervenções junto à família}

Além do paciente, lembramos que a família deverá ser atendida, dentro do possível e dependendo das possibilidades da instituição na qual o paciente é cuidado.

A importância de relações afetivas tem sido amplamente comprovada em todas as doenças e em especial no câncer. Qualquer tipo de doença afetará sempre a família de seu portador, pois quando um indivíduo adoece, a família sofre muitas vezes um desequilíbrio em sua estrutura.

Entendendo a família como um sistema, onde os diferentes componentes são afetados sempre que alguma coisa atinge um de seus membros, podemos alcançar a importância deste tipo de atendimento.

O que é observado no cotidiano da clínica é que famílias estruturadas antes do surgimento da doença em um de seus membros, adaptam-se a nova situação mais rapidamente e de maneira mais eficaz do que famílias que anteriormente já eram desestruturadas.

Nas diferentes etapas da doença, o profissional de Psico-Oncologia deve estar atento aos movimentos dos familiares, prestando um atendimento anteriormente estabelecido por um contrato, ou de acordo com o surgimento desta necessidade.

Ribeiro (1994) lembra que a família passa por diversas etapas quando um de seus membros adoece com câncer. A fase mais difícil, segundo relatos, é o diagnóstico, contribuem para isto, o impacto da notícia, a sensação de perda de controle, a impotência, a vivência de desamparo e a convicção de que receberam uma sentença de morte. Sendo que a frequência dos atendimentos fica na dependência do grau de (des)estruturação familiar e da situação clínica do paciente.

Dentro dos limites desta exposição devemos citar um aspecto que não pode ser ignorado, o lugar que o paciente ocupa na estrutura familiar. São muito diferentes as reações familiares se o paciente é um idoso, um chefe provedor, uma mãe com filhos ainda pequenos, e assim por diante.

\section{Intervenções em grupo}

Em nossa experiência, na atividade terapêutica grupal os pacientes sentem-se contidos, pertencendo a um grupo de iguais.

Na década de 70, Carl Simonton, oncologista e radioterapeuta deu início a um processo psicoterápico realizado em grupo, complementando as intervenções médicas às quais vinham se submetendo em função da doença. Ele Observou os pacientes que se submetiam a esses procedimentos tinham uma sobrevida bem maior, à medida que seguiam uma abordagem que envolvia corpo e mente. Foi um dos primeiros atendimentos em grupo para pacientes de câncer.

Resumidamente a proposta de Simonton era apresentar aos pacientes reunidos em um grupo, acompanhados de um familiar, a relação entre mente e corpo, demonstrando a importância desta relação na evolução da doença. Os pacientes deviam encontrar-se ao longo de 9 (nove) semanas durante um dia inteiro, onde era apresentado um tema relativo ao surgimento do câncer, o stress que o paciente eventualmente vivia, aos tipos de tratamentos e seus efeitos colaterais, os efeitos sobre a família, ente outros que poderiam emergir do próprio grupo. Cada encontro era iniciado por um relaxamento corporal, e uma visualização, processos baseados nas propostas de Milton Erickson, apresentadas em texto de 
Rosen (1994). O grupo tinha uma proposta educativa, e um dos objetivos propostos era de que finalizado o trabalho grupal, os pacientes deveriam continuar com os procedimentos ensinados, no sentido de autoajuda.

Posteriormente as ideias de Simonton foram questionadas sob a alegação que o embasamento teórico de tais procedimentos não obedeciam aos cânones científicos exigidos pela comunidade científica.

\section{Reflexões finais}

No Brasil o câncer é a segunda causa de morte, desde o seu diagnóstico e tratamento o paciente, seus familiares e, inclusive, os profissionais envolvidos nos cuidados desta patologia passam por sofrimentos psíquicos muito significativos. A psicologia vem ao logo dos anos criando e desenvolvendo vértices de observação e compreensão, bem como técnicas de intervenção para lidar da forma mais efetiva com esta realidade tão complexa que é a doença oncológicas e suas consequências.

\section{Referências}

Ader, R. Cohen \& N. Felten, D. (1995) Psychoneuroimmunology: interactions between the nervous system and the immune system. The Lancet. 345, 99-102.

Alves, R, Santos, G., Ferreira, P., Costa, A. \& Costa, E. (2017) Atualidades Sobre a Psicologia da Saúde e a Realidade Brasileira. Psicologia, Saúde \& Doenças, 18(2)

Bauer, M.E. (2004) Como os fatores psicológicos influenciam o surgimento e progressão do câncer? Rev. Bras. Oncologia Clínica. Vol. 1. N. ${ }^{\circ} 1$

Mandal. A. (2019) Cancer-History - Recuperado de: https://www.news-medical. net/health/Cancer-History-(Portuguese).aspx

Campos, E.M.P.; Rodrigues, A.L.; Machado, P. \& Alvarez, M. (2007). Intervenção com mães em casa de apoio. Psicol. estud., 12(3), 635-640.

Coelho, F.R.G. (1998). O Controle do Câncer. In M.M. Brentani; F.R.G. Coelho; H. Iyeyasu \& L.P. Kowalski (Orgs.) Bases da Oncologia (pp.1-22). São Paulo: LEMAR.

Deitos, T.F.H. \& Gaspary, J.P.F. (1997) Efeitos biopsicossociais e psiconeuroimunológicos do Câncer sobre pacientes e familiares. Rev. Bras. cancerol;43(2):117-26, abr.jun.

Conselho Federal de Psicologia (2016) (https://cfp.org.br/wp-content/uploads/ sites/15/2016/12/Lei-4119_1962.pdf)

Pinheiro, R (2009) Integralidade em Saúde. In: Pereira, I. B; Lima, J. C. F. Dicionário da Educação Profissional em Saúde. Recuperado de:http:// www.sites.epsjv.fiocruz.br/dicionario/verbetes/intsau.html
International Agency for Research in Cancer(2018)Recuperado de: https:// gco.iarc.fr/today/online-analysis-

Gimenes, M.G.G. (1994). O que é Psiconcologia. In M.M. Carvalho (Org.). Introdução à Psiconcologia (pp.35-56). Campinas: Editorial Psy II.

Grassi, L.; Biancosino, B.; Marmai, L.; Rossi, E. \& Sabato, S. (2007). Psychological Factors Affecting Oncology Conditions. In P. Porcelli \& N. Sonino (Orgs.) Psychological Factors Affecting Medical Conditions (pp.57-71) Switzerland: Basel.

Gorayeb, R. (2010) Psicologia da Saúde no Brasil. Psic.: Teoria e Pesquisa, (online)Vol. 26 n.spe, pp.115-122. ISSN 0102-3772. https://doi.org/10.1590/ S0102-37722010000500010.

Holland, J.C. \& Rowland, J.H. (1990). Handbook of Psychooncology: Psychological Care of the patient with cancer. New York: Oxford University Press.

Holland, J.C. (2002). History of psycho-Oncology: Overcoming attitudinal and conceptual barriers. Psychosomatic Medicine, 64, 206-221. New York: Oxford University Press

Hipócrates. (1999). Da natureza do homem (H. Cairus, trad.). História, Ciência, Saúde, Manguinhos, 6(2. (Original dos séculos IVV a.C.)

Holland, J.C. \& Rowland, J.H. (1990). Handbook of Psychooncology: Psychological Care of the patient with cancer. New York: Oxford University Press.

Instituto Nacional de Câncer (2020)- Recuperado de: https://www.inca.gov. br/estimativa/estado-capital/brasil.

Kerbauy, R. R. (2002) Comportamento e Saúde: Doenças e Desafios. Psicologia USP, v. 13, n. 1, p. 11-28.

LeShan, L. (1994). Brigando pela Vida. São Paulo: Summus.

Maia, E.M.C., Silva, N.G., Martins, R.R. \& Sebastiani, R.W. (2015) Psicologia da Saúde-Hospitalar: Da Formação a Realidade. Univ. Psychol. vol.4 no.1

Instituto Oncoguia (2020). Recuperado de: http://www.oncoguia.org.br/conteudo/cancer-avancado-metastase-e-metastase-ossea/13285/357/

Opas Brasil (2018) Recuperado de: https://www.paho.org/bra/index. php?option $=$ com_content $\&$ view $=$ article $\&$ id $=5588$ : folha-informativa -cancer\&Itemid $=1094$

Ribeiro, E.M.P. (1994) O paciente terminal e a família. In M.M.M.J. Carvalho (Org.) Resgatando o viver (pp 72-84). São Paulo: Summus.

Rosen, S. (1994) Minha voz irá contigo. Os contos didáticos de Milton Erick. Campinas: Editorial Psy.

Sebastiani, R.W., Pelicioni, M.C.F., \& Chiattone, H.B.C. (2002). Psicología de la Salud Latinoamericana. International Journal of Clinical and Health Psychology, Vol. 2, $\mathrm{N}^{\mathrm{o}} 1$

Servan-Schreiber, D. (2008). Anticancer. Rio de Janeiro: Objetiva.

Simonton, O.C.; Simonton, S.M. \& Creighton, J. (1987). Com a Vida de Novo: uma abordagem de auto-ajuda para pacientes com câncer. São Paulo: Summus Editorial.

Spink, M. (2003). Psicologia Social e Saúde. São Paulo: Editora Vozes.

Schwartsmann, G., Katz, A., Cunha, F.M., Vinholes, J., Gansl, R.C., Simon, S.D. (1991) - Oncologia clínica: princípios e prática. Porto Alegre: Artes Médicas.

Submetido em: 6-10-2020

Aceito em: 19-4-2021 